\title{
The influence of the tonometer position on canine intraocular pressure measurements using the Tonovet ${ }^{\circledR}$ rebound tonometer
}

\author{
Juliana Kravetz de Oliveira ${ }^{1, *}$, Fabiano Montiani-Ferreira ${ }^{1}$ and David L. Williams ${ }^{2}$ \\ ${ }^{1}$ Department of Veterinary Medicine, Federal University of Paraná, Rua dos Funcionários, 1540, Curitiba, Paraná, \\ Brazil \\ ${ }^{2}$ Department of Veterinary Medicine, University of Cambridge, Madingley Road, Cambridge, CB3 OES UK
}

\begin{abstract}
The objective of this study was to assess the variability of readings made using the Tonovet ${ }^{\circledR}$ rebound tonometer for measurement of intraocular pressure (IOP) in the peripheral cornea and in angulated positions on the canine corneal surface. Forty-six client-owned dogs admitted for ophthalmic evaluation at the Queen's Veterinary School Hospital, University of Cambridge were included in the study. IOP readings were taken at a variety of locations and using the tonometer at a number of different angles to the cornea: 1) Perpendicularly at center of the cornea (CC); 2) At the center of the cornea but with the tonometer positioned at four angles, and 3) At four different points on the peripheral cornea. All values were compared with the values recorded at the recommended CC position. IOP values were significantly underestimated in seven positions, with median and interquartile range from $12.1 \pm 4 \mathrm{mmHg}$ (nasal on periphery) to $15 \pm 5 \mathrm{mmHg}$ (laterally angled at center), varying between $0 \mathrm{mmHg}$ to $2.9 \mathrm{mmHg}$ from the CC value. While dorsally angled in the central cornea were not significantly different from those at $\mathrm{CC}(p=0.09)$. Median values were lower for measurements in peripheral positions when compared to angled central positions. These results demonstrate that angling the tonometer or measuring in peripheral regions can result in small but statistically significant underestimation of IOP values.
\end{abstract}

Keywords: Dog, Intraocular pressure, Peripheral tonometry, Rebound tonometry.

\section{Introduction}

Tonometry is an important method for measurement of intraocular pressure (IOP) during a complete ophthalmic evaluation in dogs. It is important for the diagnosis and control of glaucoma as well as uveitis. Glaucoma is a pathological increase in IOP that causes damage to the optic nerve and retina resulting in blindness. Accurate IOP measurement is crucial to determine the therapeutic approach, since IOP abnormalities can cause significant morbidity (Renwick and Petersen-Jones, 2009; Plummer et al., 2013).

The rebound tonometer is a portable tonometer with a small probe, its use is increasing in veterinary medicine because it is well tolerated by most animals (Kontiola et al., 2001; Prashar et al., 2007), and does not require topical anesthesia. Additionally, it is the most accurate hand-held tonometer (Tofflemire et al., 2017) and can provide accurate readings even in inexperienced hands (Abraham et al., 2008; Sahin et al., 2008).

The technique of rebound tonometry was first reported in laboratory animals in the early 2000's (Kontiola et al., 2001; Danias et al., 2003) and its use became widespread in medical (Abraham et al., 2008; Muttuvelu et al., 2012; Dosunmu, et al., 2014) and veterinary (Reuter et al., 2010; Rusanen et al., 2010;
Nagata et al., 2011; Selleri et al., 2012; Slack et al., 2012; Thompson-Hom and Gerding, 2012; Zhang et al., 2014;) ophthalmology in subsequent years.

The Tonovet ${ }^{\circledR}$ tonometer (Tiolat Ltd., Helsinki, Finland) uses a small magnetized probe, which is directed toward the corneal surface by an electric coil. After impacting the cornea, the deceleration of rebound is measured by the induced voltage in the sensitive coil (Kontiola, 1997; Kontiola, et al., 2001). The manufacturer recommends that the tonometer probe is kept in a horizontal position during measurement of IOP, preventing gravitational forces affecting the speed and deceleration of the probe. However, this is not always strictly followed by every veterinary ophthalmologist. The effect of probe position on the corneal surface has been investigated in human patients (González-Méijome et al., 2006; Queirós et al., 2007; Yamashita et al, 2011; Muttuvelu et al., 2012; Beasley et al., 2013) and statistically significant differences in IOP were found when IOP was measured at the periphery of the cornea and compared to the central cornea. These investigations also have shown that IOP may be significantly underestimated when measured with the probe in an angled positions (Muttuvelu et al., 2012; Beasley et al., 2013). Only one similar study was carried in dogs (Von Spiessen et al., 2013) using the 
probe in a number of angled positions as well as in peripheral corneal locations. When tonometry was performed using angled positions but at the center of the cornea IOP data was underestimated by up to $6.5 \mathrm{mmHg}$, whereas off-center probe positioning provided a significant overestimation of $0.9 \mathrm{mmHg}$. The aim of this study was to expand this type of investigation in dogs, as anecdotal evidences did not suggest dramatic effects on IOP values when different probe positions are used. The current study evaluated potential differences of IOP values for the canine eye using a Tonovet ${ }^{\circledR}$ tonometer with the probe positioned in the periphery and in angulated positions, were compared with IOP values obtained using the recommended perpendicular position in the center of the cornea. We also aimed to evaluate the repeatability of the findings already published in the literature.

This assessment is essential since in veterinary medicine tonometry is typically performed in unsedated dogs whose globes are in constant motion, resulting in a potential misalignment of the tonometer probe in relation to the central position of the cornea. In addition, the presence of central corneal abnormalities may sometimes impair accurate tonometry measurements at this location (Von Spiessen et al., 2015). For all these reasons, it is important to know if IOP values taken in a position not perpendicular to the corneal surface or at the periphery of the cornea are significantly different from those taken perpendicularly in the center of the cornea.

\section{Materials and Methods}

Forty-six client-owned dogs undergoing ophthalmic evaluation at the Queen's Veterinary School Hospital, University of Cambridge were included in the study. All included animals were quiet and easily restrained in a sitting position, allowing the evaluation of IOP with minimal physical restraint. The study was approved by the Ethics Committee of the Department of Veterinary Medicine, University of Cambridge. A consent form was signed by each owner. The welfare of the animals was not compromised by the repeated measurements required in this study since rebound tonometry requires momentary contact with the cornea and does not cause irritation or damage. A routine ophthalmic examination was performed in each case, including: examination of cornea, anterior chamber, iris, and lens performed by direct and indirect ophthalmoscopy (Keeler practitioner direct ophthalmoscope and Vantage indirect ophthalmoscope, Windsor UK) and slit-lamp biomicroscopy (Kowa ${ }^{\circledR}$, Kowa Company ${ }^{\circledR}$, Japan) to exclude animals with corneal disease.

The IOP measurements were performed with a calibrated rebound tonometer Tonovet ${ }^{\circledR}$ positioned on the center of the cornea and in eight different positions, as shown in Figure 1, at a distance of 5 to $10 \mathrm{~mm}$ from the cornea.

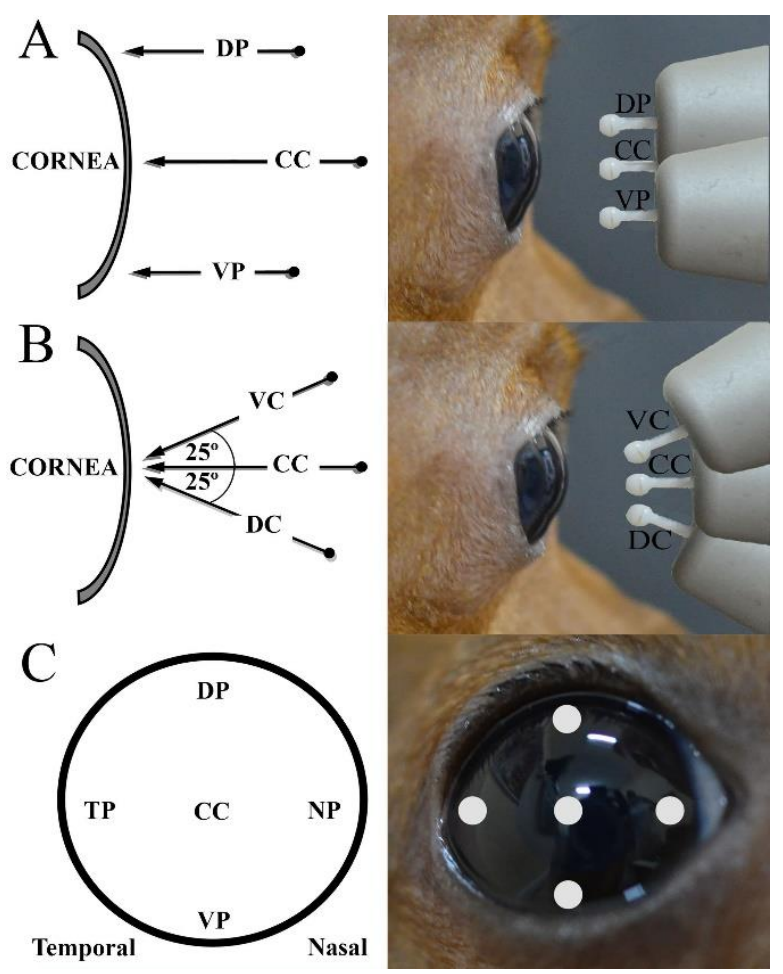

Fig. 1. The positions performed using rebound tonometer Tonovet ${ }^{\circledR}$ onto the dogs' corneal surface. A: perpendicular view of peripheral positions, showing relocation from central cornea (CC) as dorsal peripheral (DP) and ventral peripheral (VP). B: perpendicular view of angled positions, ventrally to the center (VC) and dorsally to the center (DC). C: frontal view of the peripheral positions showing relocation from central cornea (CC) as dorsal (DP), ventral (VP), nasal (NP), and temporal (TP).

The rebound tonometer has a manufacturer's calibration of ' $\mathrm{d}$ ' for use in dogs and cats. The " $\mathrm{d}$ " calibration setting was used in throughout this study. The tonometer makes six IOP readings for each evaluation. The result given by the instrument's internal processor is a mean of these values, discarding the lower and the higher result. The values recorded in this study were those with a steady display in the instrument window (ie those with a small variation between readings). Additionally, the tonometer shows an "error" signal when it measures discrepant values and it discards these automatically. A single veterinary ophthalmologist performed all the measurements, as well as all the ophthalmic evaluations.

Measurements of IOP were performed first in the central cornea (CC). Next, the tonometer probe was displaced at 20 to 25 degrees from the $\mathrm{CC}$, the angled positions being lateral to the center (LC), medial to the center (MC), dorsal to the center (DC) and ventral to the center (VC). The peripheral positions were temporal (TP), nasal (NP) dorsal (DP) and ventral (VP), and were obtained by displacing the tonometer $3 \mathrm{~mm}$ from the limbus, as shown in Figure 1. Measurements 
were made in the same order in each eye: $\mathrm{CC}$, then the angled positions VC, DC, LC and MC; followed by VP, DP, NP and TP.

The IOP values collected from right eyes of the dogs were analyzed. Median and interquartile range were calculated for variables for each position. Mean and standard deviation were calculated for age of the animals. A box-plot graph was created using Excel® (Microsoft Corporation, Washington, USA). Ninetyfive percent limits of agreement were calculated according to Bland and Altman (1986) (mean difference \pm 1.96 standard deviation of the differences) for differences between misaligned positions and central cornea. A Shapiro-Wilk normality test showed that the data did not follow a Gaussian distribution. A Friedman's test with Conover and Holm-Bonferroni post-hoc tests was used. $P<0.01$ were considered significant. Spearman correlation coefficients $(\rho)$ and determination coefficient $\left(\mathrm{R}^{2}\right)$ were calculated and simple linear regressions were applied in order to summarize and analyze possible relations between IOP values performed at $\mathrm{CC}$ and each different corneal position or probe angle. Moreover, Spearman correlation were used for comparison between both eyes of same dog. The software used for statistical analyses was StatView® 5.0 (SAS Institute Inc., Cary, North Carolina, USA).

\section{Results}

The age of the dogs examined ranged from 6 months to 15 years, with a mean of 6.1 years and standard deviation of \pm 3.55 . The animals comprised 24 female dogs and 22 males, with a total of 79 eyes. Table 1 details additional information concerning all dogs investigated. Twenty-one different breeds were represented. Mixed-breed dogs and West White Highland Terrier appeared most frequently, from which 21 globes were evaluated. Other common breeds were English cocker spaniel, Greyhound, Golden Retriever, Labrador Retriever and Jack Russell terrier.

Table 1. Summary of $46 \mathrm{dogs}$ evaluated with information about age and sex and descriptive statistics (mean and standard deviation) of intraocular pressure measurements obtained with a rebound tonometer (Tonovet $\left.{ }^{\circledR}\right)$. IOP = intraocular pressure. $\mathrm{CC}=$ central cornea.

\begin{tabular}{lccc}
\hline \multirow{2}{*}{ Sex } & Number & \multicolumn{2}{c}{ Age (Years) } \\
\cline { 3 - 4 } & & $\begin{array}{c}\text { Minimum and } \\
\text { maximum }\end{array}$ & $\begin{array}{c}\text { Mean } \pm \text { standard } \\
\text { deviation }\end{array}$ \\
\hline Males & 22 & $0.5-13$ & $6.1 \pm 3.73$ \\
Females & 24 & $0.6-15$ & $6.1 \pm 3.45$ \\
Total & 46 & $0.5-15$ & $6.1 \pm 3.55$ \\
\hline
\end{tabular}

Table 2 shows median and interquartile ranges for IOP values in each position and $95 \%$ limits of agreement. Additionally, Spearman correlation coefficient $(\rho)$ and $p$-values obtained by Friedman's test of the IOP values for each position (MC, LC, DC, VC, DP, VP, TP, NP) compared with the IOP obtained at $\mathrm{CC}$ using the rebound tonometer Tonovet $\AA$.

Statistical analysis performed by Friedman`s test with Conover post-hoc analysis and Holm-Bonferroni adjustment $(p<0.01)$ showed significant underestimation of IOP in seven positions (MC, LC, VC, DP, VP, TP, NP) when compared to CC. The only position with no statistically difference when compared to $\mathrm{CC}$ was DC $(p=0.09)$.

Peripheral positions had lower median values than angled positions. The highest Spearman correlation coefficient $(\rho)$ values was LC $(\rho=0.49, p<0.01)$ and the lowest was the peripheral position NP $(\rho=0.09, p$ $=0.5$ ).

Correlation between eyes of the same dog were statistically significant $(\rho=0.24, p=<0.01)$.

Figure 2 shows the dispersion graphs with the determination coefficient $\left(\mathrm{R}^{2}\right)$ for each position in comparison with $\mathrm{CC}$, the which the values were 0.05 (DC), 0.11 (VC), 0.24 (MC) and 0.27 (LC) for angled positions and 0.02 (NP), 0.03 (TP), 0.03 (DP) and 0.03 (VP) for peripheral positions.
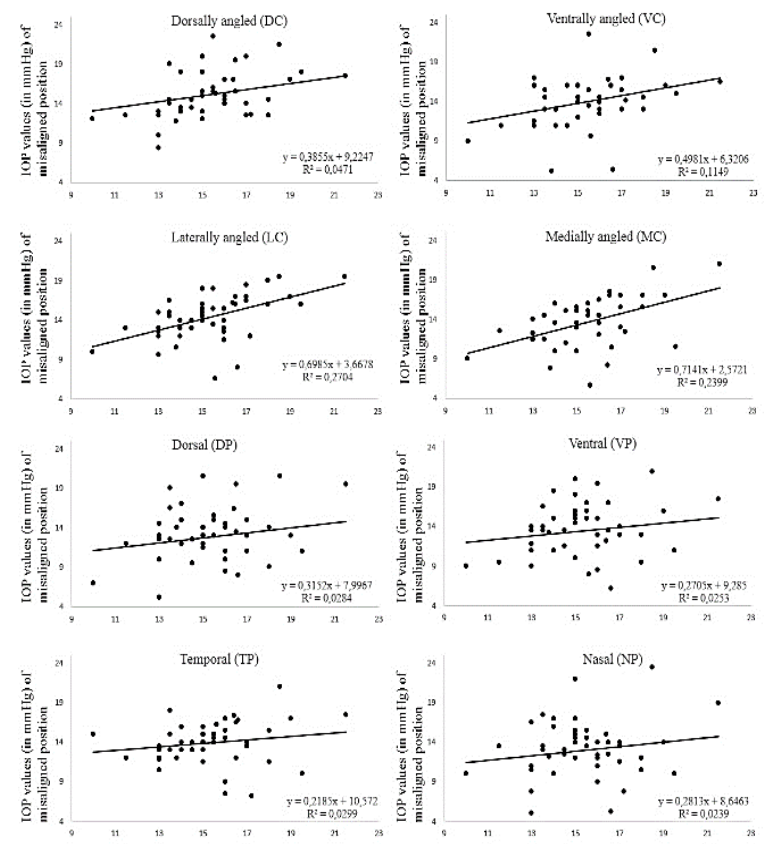

Fig. 2. Dispersion graphs for each position of measurement of IOP with the rebound tonometer Tonovet ${ }^{\circledR}$ compared with the CC. For every position there is a positive correlation. The solid line represents the $\mathrm{CC}$ position and dashed line represents the position indicated in the legend. 
Table 2. Statistics (median and interquartile range) relating to intraocular pressure measurements obtained with a rebound tonometer (Tonovet $\left.{ }^{\circledR}\right)$ in different positions on the cornea compared with central cornea $(\mathrm{CC}) . P$ values generated by Friedman`s test with Conover post-hoc analysis and adjusted by Holm-Bonferroni method in comparison with CC. Spearman correlation coefficient $(\rho)$ and $95 \%$ limits of agreement.

\begin{tabular}{lcccc}
\hline \multicolumn{1}{c}{ Probe position } & $\begin{array}{c}\text { Median } \pm \\
\text { interquartile range }\end{array}$ & $p$ values & $\begin{array}{c}\text { Limit of agreement of } \\
95 \% \text { in } \mathrm{mmHg}\end{array}$ & $\begin{array}{c}\text { Spearman correlation } \\
\text { coefficient }(\rho)\end{array}$ \\
\hline Central cornea (CC) & $15 \pm 3$ & - & - & - \\
\hline Angled ventrally to the center (VC) & $14 \pm 4.5$ & $<0.01$ & {$[-5.4 ; 8.4]$} & 0.39 \\
Angled dorsally to the center (DC) & $15 \pm 5.3$ & 0.09 & {$[-8.1 ; 8.75]$} & 0.31 \\
Angled laterally to the center (LC) & $15 \pm 5.3$ & $<0.01$ & {$[-4.8 ; 7.0]$} & 0.49 \\
Angled medially to the center (MC) & $14 \pm 4$ & $<0.01$ & {$[-4.5 ; 8]$} & 0.48 \\
Dorsal position (DP) & $13 \pm 3.75$ & $<0.01$ & {$[-6.5 ; 11.8]$} & 0.27 \\
Ventral position (VP) & $14 \pm 3$ & $<0.01$ & {$[-5.9 ; 11.6]$} & 0.1 \\
Temporal position (TP) & $13.6 \pm 5$ & $<0.01$ & {$[-7.1 ; 11.1]$} & 0.28 \\
Nasal position (NP) & $12.1 \pm 4$ & $<0.01$ & {$[-6.5 ; 11.8]$} & 0.09 \\
\hline
\end{tabular}

A weak positive correlation is observed between offcenter/angled readings and central corneal readings. The $\mathrm{R}^{2}$ values for these regression lines are close to zero for most locations. Only the medially and laterally angled values are $\mathrm{R}^{2}$ values for these slopes marginally $>0.2$, which for a clinical measurement is hardly indicative of a strong association.

Figure 3 shows the Bland-Altman plots for each position, with a $95 \%$ interval of agreement, the limits values for angled positions (in $\mathrm{mmHg}$ ) were approximately \pm 6.9 (VC), \pm 8.4 (DC), \pm 5.9 (LC) and \pm 6.2 (MC). The peripheral positions had larger limits with $95 \%$ of agreement, which were (in $\mathrm{mmHg}) \pm 9.2$ (DP), $\pm 7.1(\mathrm{VP}), \pm 9.1$ (TP) and $\pm 8.73(\mathrm{NP})$. Figure 4 shows box-plot graphs of the IOPs values in $\mathrm{mmHg}$, obtained by the comparison of misaligned positions with CC, including outlier's values.

\section{Discussion}

The misplacement of the rebound tonometer probe during measurements could lead to both underestimated and overestimated IOP values, being imprecise and inaccurate and could markedly differ from values obtained on $\mathrm{CC}$ in an unpredictable manner. Three positions off-angle (VC, LC and MC) and four positions off-axis (DP, NP, TP and VP) showed significant statistical differences from $\mathrm{CC}$. The only position in which IOP measurements showed no significant difference from CC was DC.

The use of rebound tonometer is becoming increasingly popular in veterinary medicine due to its practicality and accuracy when compared to manometry (Knollinger et al., 2005; McLellan et al., 2013; Ma et al., 2016; Tofflemire et al., 2017), which is an invasive method that documents true IOP values. One of the limitations of this study is that true IOP values given by manometry were not available at the data collection. Nevertheless our research question was whether there is a significant difference between the standard result and that obtained when the probe is positioned angled or peri-centrally and this does not require determination of the true IOP.

This study was designed to assess whether misalignment of rebound tonometer can result in increased or decreased values of IOP. To find such results, rebound tonometry was repeated in each position on the corneal surface. No disturbance or damage to the cornea was observed. However, it is well known that some applanation tonometers produce the tonographic effect, which is a phenomenon in which IOP values decline with repeated tonometry (Stocker, 1958; Moses, 1961; Krakau and Wilke, 1971; Gaton et al., 2010; Zimmermann et al., 2017). In a study of repeated rebound tonometry in children, the tonographic effect did not occur (Dosunmu et al., 2014).

However, in mice, repeated rebound tonometry resulted in a significant reduction of IOP readings, a reduction of $2 \mathrm{mmHg}$ after 10 measurements (Morris et al., 2006). To our knowledge, there is no information about IOP values changes in dogs due to repeated measurements with rebound tonometry. Thus, we cannot discard the possibility that the tonographic effect occurred in this study, and this would have particularly affected measurements made in the peripheral positions since these were evaluated last in every case and these values did consistently underestimate IOP. Further studies are needed to investigate the tonographic effect with rebound tonometry in canine corneas.

Correlation of IOP values in angulated misaligned positions with $\mathrm{CC}$ were positive and statistically significant, however with a low strength, which can be due to the small variability of IOP values, which only $\mathrm{CC}$ values between 15 and $25 \mathrm{mmHg}$ were accepted for this study. Thus, if uveitic and glaucomatous eyes were analyzed, stronger correlation will probably be seen. 


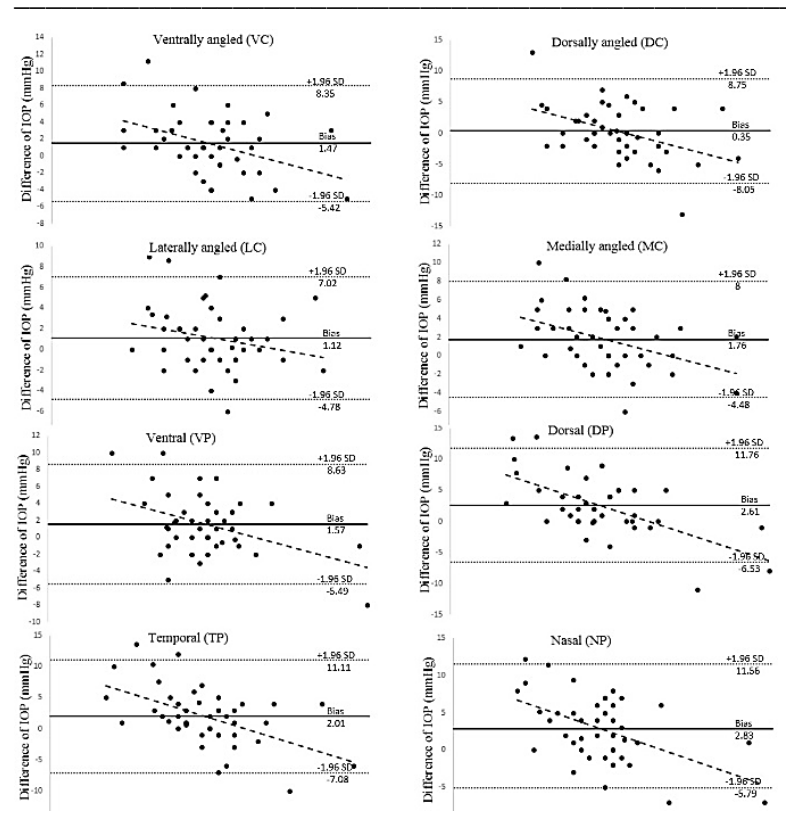

Fig. 3. Bland-Altman plots showing the lack of agreement of IOP values measured by rebound tonometer (Tonovet ${ }^{\circledR}$ ) in different positions compared with CC. Vertical axis shows the difference in IOP reading versus the average $\mathrm{CC}$ and corresponding misaligned position value on horizontal axis. The dashed lines shows the limits of agreement of $95 \%$. The full line is representative of the means difference and the dotted line is the linear regression with the equation and $\mathrm{R}^{2}$, determination coefficient values.

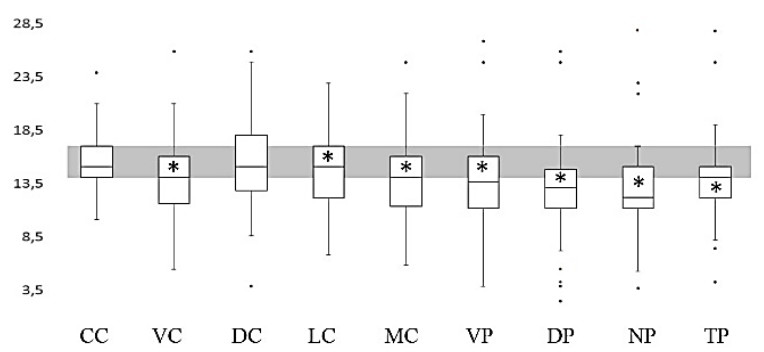

Fig. 4. Box plot graph of intraocular pressure values measured by rebound tonometer (Tonovet $\AA$ ) in different positions on corneal surface. * Shows the positions with statistically significant differences compared to $\mathrm{CC}$.

For comparison, the dispersion graphs also show the positive correlation, but due to the small variability values, determination coefficients were also low. Nevertheless, peripheral positions had no statistically significant correlation, showing that in these positions, IOP values will not co-vary similar with $\mathrm{CC}$, given results may indicate that peripheral readings undergo more variables than angulated positions. Additionally, the Bland-Altman graphs show both underestimation and overestimation of values found in misaligned positions compared to $\mathrm{CC}$.

Meanwhile box-splot graph shows that interquartile range were below $\mathrm{CC}$, showing the tendency for underestimation. In short, the findings showed that rebound tonometry in all misaligned positions could give unreliable results. Additionally, the wider range of limits of agreement for the peripheral positions in Bland-Altman plots, and lowers values of Spearman correlation and determination coefficients means that the rebound tonometry measurements in these positions were less robust.

Von Spiessen et al. (2013) also found a tendency for underestimated values of IOP in dogs when the tonometer was in an angled position. In human patients, $10^{\circ}$ degrees of angulation can result in a statistically significant underestimation of IOP readings (Beasley $e t$ al., 2013), however this effect was not seen in rats with rebound tonometer Tonolab $\AA$, similar to Tonovet $\AA$, used in an angled position of $25^{\circ}$ degrees (Kontiola et al., 2001). Rebound tonometry calculates IOP based on parameters of movement; such as the time spent in contact with the cornea, return velocity and deceleration. The last parameter is intimately correlated to IOP (Kontiola, 1997). According to Newton`s third law (action-reaction principle) when a body exerts a force on a second body, simultaneously, the second body exerts an equal and opposite force (Newton and Motte, 2016). Force is a vector quantity and depends on mass and acceleration ( $\mathrm{F}=$ mass $*$ acceleration). In the case of rebound tonometry, the deceleration of the probe when contacting the corneal surface will be one of the main determining factors for the IOP result, since the mass of the probe remains constant. When the probe is applied at an angle, the force will be distributed according to vectors, and consequently, the IOP values will be lower. The equation used in mechanics can be extrapolated to give the resultant force at a determined angle and can be written as $\mathrm{F}_{\text {angled position }}=\mathrm{F}_{\text {central cornea }}$ * Cosine (angle), in which cosine will be always be $<1$, resulting in a lower IOP. This equation is for a hypothetical environment without considering the effects of variables such as gravity, air resistance or probe slippage, nevertheless it gives an approximate IOP value for any determined angle.

Interestingly, the only position in which there was no significant difference compared with CC was DC. IOP measurements are lower at DC than $\mathrm{CC}$, as would be expected in angled positions; however, in the DC position gravity facilitates the probe's return to its original position. Another possibility that DC may not be significantly different presumably due to their considerable variability. Although there was no significant difference, this position is also not recommended for measurement of IOP, due to interference of variables, especially gravity, which were not considered by the tonometer during the calculations of IOP value.

There was thus some degree of agreement between the results of Von Spiessen et al. (2013) and the results of 
our investigation. In both studies, angled positions have a tendency to underestimate IOP. The difference was however, lower in our study. This disparity between the studies may be due to differences in the positions investigated, since Von Spiessen et al. (2013) assessed only two angulations (dorsally and ventrally), whereas our study investigated laterally and medially angled as well, providing more results with less interference of gravity.

The IOP results obtained in peripheral positions in this study are in disagreement with the values found by Von Spiessen et al. (2013) in which the values of IOP in these locations were overestimated. Our results show an underestimation of IOP when measurements were made in the peripheral cornea, a similar finding to results previously reported in human medicine (González-Méijome et al., 2006; Queirós et al., 2007; Muttuvellu et al., 2012).

It is well known that corneal thickness has a positive correlation with IOP values measured by rebound tonometry in different species, including dogs (Martinez-de-la-Casa et al., 2005; Prashar et al., 2007; Chui et al., 2008; Harada et al., 2008; Sahin et al, 2008; Poostchi et al., 2009; Park et al., 2011, 2013; Rao et al., 2014).

The cornea is thicker peripherally than centrally (Gilger et al., 1991; Strom et al., 2016), however in paraxial positions there was no significant difference from central corneal thickness (Strom et al., 2016). In our study, measurement was made at a distance of $3 \mathrm{~mm}$ from the limbus, whereas Von Spiessen et al. (2013) made measurements at approximately $1.5 \mathrm{~mm}$ from the limbus. This difference in distance might possibly explain the disparity of IOP values in both studies, since the cornea is thicker nearer the limbus.

However, the increase in thickness of the peripheral cornea does not necessarily mean an increase of hardness. The distribution of collagen fibers can change corneal elasticity, and these are more compressed in the central cornea than in the periphery in dogs (Nagayasu et al., 2009). Similar changes in collagen distribution have been reported in man (Boote et al., 2003). The underestimated IOP values found in peripheral positions in this study resembles the studies in human eyes (González-Méijome et al., 2006; Queirós et al., 2007; Muttuvelu et al., 2012).

The underestimation of IOP values in peripheral positions may be a due to greater elasticity of peripheral cornea, resulting in a smaller deceleration of the rebound tonometer probe and, consequently, a lower value of IOP. Conversely, Yamashita et al. (2011) found overestimation of IOP in peripheral positions in the human eye; however the experimenters asked the patients to move their eyes to make the measurements, and this can apply tensile forces to the cornea due to extraocular muscles movements.
The underestimated IOP values found in the current study in peripheral positions could also be a result of the angled position of the tonometer assumed due to natural corneal curvature. The values obtained show that the rebound tonometer measurements are somewhat less robust in peripheral positions, as previously shown in a study in chickens (Prashar et al., 2007), which also showed weaker positive correlation with CC and a wider limit of agreement in BlandAltman analysis.

Besides central corneal thickness, there are other corneal biomechanical properties that may influence IOP values measured by tonometry (Liu and Roberts, 2005), such as hysteresis, corneal resistance factor (Liu and Roberts, 2005; Chui et al., 2008; Ogbuehi and Osuagwu, 2014; Deol et al., 2015), corneal curvature (Matsumoto et al., 2000; Liu and Roberts, 2005; Harada et al., 2008) and pre-corneal tear film (Zeng et al., 2008). These biomechanical features may have a greater influence than thickness on IOP readings in the central cornea and have a significant correlation with IOP values measured by rebound tonometry (Chui et al., 2008; Deol et al., 2015). All of these properties should be considered during IOP investigations in dogs, given that they may markedly reduce or increase the measured value of IOP. However, investigating such factors is difficult and costly given the complex equipment required, this probably explains the lack of validated studies of corneal biomechanical properties in dogs.

Although an underestimation tendency were found, rebound tonometry in misaligned positions also resulted in overestimated values in both angled and peripheral positions. These findings can be the result of increased deceleration of probe rebound on cornea, giving higher IOP values. Potential reasons for this were variations on individual corneal biomechanical properties and the presence of tensile forces on cornea by extra ocular muscles (Yamashita et al., 2011; Von Spiessen et al., 2013).

Correlation between eyes of the same dog were statistically significant, showing that in the same dog the resulting values of both eyes were similar. Which can due to individual variations of anatomy and corneal biomechanical proprieties, shared by both eyes of the same dog, which will influence IOP readings.

\section{Conclusion}

The present study has shown that there are small, but significant, differences between TonoVet ${ }^{\circledR}$ measurements taken in different corneal positions and angles, with a tendency for underestimation. Misaligned positions can differ from CC markedly and unpredictably, giving underestimated and overestimated values.

\section{Conflict of interest}

The authors declare that there is no conflict of interests. 


\section{References}

Abraham, L.M., Epasinghe, N.C.R., Selva, D. and Casson, R. 2008. Comparison of the ICare ${ }^{\circledR}$ rebound tonometer with the Goldmann applanation tonometer by experienced and inexperienced tonometrists. Eye 22, 503-506.

Beasley, I.G., Laughton, D.S., Coldrick, B.J., Drew, T.E., Sallah, M. and Davies, L.N. 2013. Does rebound tonometry probe misalignment modify intraocular pressure measurements in human eyes? J. Ophthalmol. doi: 10.1155/2013/791084.

Bland, J.M. and Altman, D.G. 1986. Statistical methods for assessing agreement between two methods of clinical measurement. The Lancet 8, 307-310.

Boote, C., Dennis, S., Newton, R.H., Puri, H. and Meek, K.M. 2003. Collagen fibrils appear more closely packed in the prepupillary cornea: optical and biomechanical implications. Invest. Ophthalmol. Vis. Sci. 44, 2941-2948.

Chui, W., Lam, A., Chen, D. and Chiu, R. 2008. The influence of corneal properties on Rebound Tonometry. Ophthalmology 115, 80-84.

Danias, J., Kontiola, A.I., Filippopoulos, T. and Mittag, T. 2003. Method for the noninvasive measurement of intraocular pressure in mice. Invest. Ophthalmol. Vis. Sci. 44, 1138-1141.

Deol, M., Taylor, D.A. and Radcliffe, N.M. 2015. Corneal hysteresis and its relevance to glaucoma. Curr. Opin. Ophthalmol. 26, 96-102.

Dosunmu, E.O., Marcus, I., Tung, I., Thiamthat, W. and Freedman, S.F. 2014. The effect of repeated measurements and the use of topical anesthetic on rebound tonometry values in children. J. AAPOS. 18, 619-621.

Gaton, D.D., Ehrenberg, M., Lusky M., Wussuki-Lior, O., Dotan, G., Weinberger, D. and Snir, D. 2010. Effect of repeated applanation tonometry on the accuracy of intraocular pressure measurements. Curr. Eye Res. 35: 475-479.

Gilger, B.C., Whitley, R.D., Mclaughlin, S.A., Wright, J.C. and Drane, J.W. 1991. Canine corneal thickness measured by ultrasonic pachymetry. Am. J. Vet. Res. 52, 1570-1572.

González-Méijome, J.M., Jorge, J., Queirós, A., Fernandes, P., Montés-Micó, R., Almeida, J.B. and Parafita, M.A. 2006. Age differences in central and peripheral intraocular pressure using a rebound tonometer. Br. J. Ophthalmol. 90(12), 1495-1500.

Harada, Y., Hirose, N., Kubota, T. and Tawara, A. 2008. The influence of central corneal thickness and corneal curvature radius on the intraocular pressure as measured by different tonometers: noncontact and Goldmann aplanation tonometers. J. Glaucoma 17, 619-625.

Knollinger, A.M., La Croix, N.C., Barrett, P.M. and Miller, P.E. 2005. Evaluation of a rebound tonometer for measuring intraocular pressure in dogs and horses. J. Am. Vet. Med. Assoc. 227(2), 244-248.

Kontiola, A.I., Goldblum, D., Mittag, T. and Danias, J. 2001. The induction/impact tonometer: a new instrument to measure intraocular pressure in the rat. Exp. Eye Res. 73, 781-785.

Kontiola, A. 1997. A new electromechanical method for measuring intraocular pressure. Doc. Ophthalmol. 93, 265-276.

Krakau, C.E. and Wilke, K. 1971. On repeated tonometry. Acta Ophthalmol. (Copenh). 49(4), 611614.

Liu, J. and Roberts, C.J. 2005. Influence of corneal biomechanical properties on intraocular pressure measurement. Quantitative analysis. J. Cataract Refract. Surg. 31, 146-155.

Ma, D., Chen, C.B., Liang, J., Lu, Z., Chen, H. and Zhang, M. 2016. Repeatability, reproducibility and agreement of intraocular pressure measurement in rabbits by the Tonovet and Tono-pen. Sci. Rep. 6:35187. doi: 10.1038/srep35187.

Martinez-de-la-Casa, J.M., Carcia-Feijoo, J., Castillo, A. and Garcia-Sanchez, J. 2005. Reproducibility and clinical evaluation of rebound tonometry. Invest. Ophthalmol. Vis. Sci. 46, 4578-4580.

Matsumoto, T., Makino, H., Uozato, H., Saishin, M. and Miyamoto, S. 2000. The influence of corneal thickness and curvature on the difference between intraocular pressure measurements obtained with a Non-contact tonometer and those with a Goldmann applanation tonometer. Nippon. Ganka. Gakkai. Zasshi. 104(5), 317-323.

McLellan, G.J., Kemmerling, J.P. and Kiland, J.A. 2013. Validation of the Tonovet $\AA$ rebound tonometer in normal and glaucomatous cats. Vet. Ophthalmol. 16(2), 111-118.

Morris, C.A., Crowston, J.G., Lindsey, J.D., Danias, J. and Weireb, R.N. 2006. Comparison of invasive and non-invasive tonometry in mouse. Exp. Eye Res. 82(6), 1094-1099.

Moses, R.A. 1961. Repeated applanation tonometry. Ophthalmologica 142(6), 663-668.

Muttuvelu, D.V., Baggesen, K. and Ehlers, N. 2012. Precision and accuracy of the ICare tonometer Peripheral and central IOP measurements by rebound tonometry. Acta Ophthalmol. 90, 322-326.

Nagata, N., Yuki, M. and Hasegawa, T. 2011. In vitro and in vivo comparison of applanation tonometry and rebound tonometry in dogs. J. Vet. Med. Sci. 73, 1585-1589.

Nagayasu, A., Hirayanagi, T., Tanaka, Y., Tangkawattana, P., Ueda, H. and Takehana, K. 2009. Site-Dependent differences in collagen lamellae in the corneal substantia propria of Beagle dogs. J. Vet. Med. Sci. 71, 1229-1231. 
Newton, I. and Motte, A. 2016. Philosophiae naturalis principia mathematica. (English). Createspace Independent Publishing Plataform, 500.

Ogbuehi K.C. and Osuagwu, U.L. 2014. Corneal biomechanical properties: precision and influence on tonometry. Cont. Lens Anterior Eye 37(3), 124131.

Park, Y.W., Jeong, M.B., Lee, E.R., Lee, Y., Ahn, J.S., Kim, S.H. and Seo, K. 2013. Acute changes in central corneal thickness according to experimental adjustament of intraocular pressure in normal canine eyes. J. Vet. Med. Sci. 75, 1479-1483.

Park, Y.W., Jeong, M.B., Kim, T.H., Ahn, J.S., Ahn, J.T., Park, S.A., Kim, S.E. and Seo, K. 2011. Effect of central corneal thickness on intraocular pressure with the rebound tonometer and the applanation tonometer in normal dogs. Vet. Ophthalmol. 14, 169-173.

Plummer, C.E., Regnier, A. and Gelatt, K.N. 2013. The canine glaucomas. In: Gelatt, K.N; Gilger, B.C; Kern, T.J. Veterinary Ophthalmology. $5^{\text {th }}$ edition. John Wiley and Sons, Inc, Hoboken (NK), pp: 1050-1145.

Poostchi, A., Mitchell, R., Nicholas, S., Purdie, G. and Wells, A. 2009. The iCare rebound tonometer: comparisons with Goldmann tonometry, and influence of central corneal thickness. Clin. Exp. Ophthalmol. 37, 687-691.

Prashar, A., Guggenheim, J.A., Erichsen, J.T., Hocking, P.M. and Morgan, J.E. 2007. Mearusement of intraocular pressure (IOP) in chickens using a rebound tonometer: quantitative evaluation of variance due to position inaccuracies. Exp. Eye Res. 85(4), 563-571.

Queirós, A., González-Méijome, J.M., Fernandes, P., Jorge, J., Montés-Micó, R., Almeida, J.B. and Parafita, M.A. 2007. Technical note: A comparison of central and peripheral intraocular pressure using rebound tonometry. Ophthalmic. Physiol. Opt. 27, 506-511.

Rao, A., Kumar, M.B.P. and Varshney, G. 2014. Relationship of Central Corneal Thickness and Intraocular Pressure by iCare Rebound Tonometer. J. Glaucoma 23, 380-384.

Renwick, P.W. and Petersen-Jones, S.M. 2009. Orbital and ocular pain. In: Peiffer, R; Petersen-Jones SM. Small Animal Ophthalmology. A Problem-Oriented Approach. $4^{\text {th }}$ Edition. Phipadelphia. Saunders Elsevier, pp: 227.

Reuter, A., Müller, K., Arndt, G. and Eule, J.C. 2010. Accuracy and reproducibility of the Tonovet rebound tonometer in birds of prey. Vet. Ophthalmol. 13, 80-85.

Rusanen, E., Florin, M., Hässig, M. and Spiess, M. 2010. Evaluation of rebound tonometer (Tonovet) in clinically normal cat eyes. Vet. Ophthalmol. 13, 31-36.

Sahin, A., Basmak, H. and Yildirim, N. 2008. The influence of central corneal thickness and corneal curvature on intraocular pressure measured by tonopen and rebound tonometer in children. $J$. Glaucoma 17, 57-61.

Selleri, P., Di Girolamo, N., Andreani, V., Guanalini, A. and D'Anna, N. 2012. Evaluation of intraocular pressure in conscious Hermann's tortoises (Testudo hermanni) by means of rebound tonometry. Am. J. Vet. Res. 73, 1807-1812.

Slack, J.M., Stiles, J. and Moore, G.E. 2012. Comparison of a rebound tonometer with an applanation tonometer in dogs with glaucoma. Vet. Rec. 171, 373. doi: 10.1136/vr.100967.

Stocker, F.W. 1958. On changes in intraocular pressure after application of the tonometer; in the same eye and in the other eye. Am. J. Ophthalmol. 45(2), 192196.

Strom, A.R., Cortés, D.E., Rasmussen, C.A., Thomasy, S.M., McIntyre, K., Lee, S., Kass, P.H., Mannis, M.J. and Murphy, C.J. 2016. In vivo evaluation of the cornea and conjunctiva of the normal laboratory beagle using time- and Fourier-domain optical coherence tomography and ultrasound pachymetry. Vet. Ophthalmol. 19, 50-56.

Thompson-Hom, A.L. and Gerding, P.A.Jr. 2012. Evaluation of intraocular pressure measurements obtained by use of a rebound tonometer and applanation tonometer in dogs before and after elective phacoemulsification. Am. J. Vet. Res. 73, 709-713.

Tofflemire, K.L., Wang, C., Jens, J.K., Ellinwood, N.M., Whitley, R.D. and Ben-Shlomo, G. 2017. Evaluation of three hand-held tonometers in normal canine eyes. Vet. J. 224, 7-10.

Von Spiessen, L. Karck, J., Rohn, K. and MeyerLindenberg, A. 2013. Clinical evaluation of the Tonovet ${ }^{\circledR}$ rebound tonometer in $\operatorname{dogs}$ and cats considering potential errors in handling. Tierarztl. Prax. Ausg. K. Kleintiere. Heimtiere. 41(4), 213220.

Von Spiessen, L., Karck, J., Rohn, K. and MeyerLindenberg, A. 2015. Clinical comparison of the TonoVet ${ }^{\circledR}$ rebound tonometer and the Tono-Pen Vet ${ }^{\circledR}$ applanation tonometer in dogs and cats with ocular disease: glaucoma or corneal pathology. Vet. Ophthalmol. 18, 20-27.

Yamashita, T., Miki, A., Ieki, Y., Kiryu, J., Yaoeda, K. and Shirakashi, M. 2011. Central and peripheral intraocular pressure measured by a rebound tonometer. Clin. Ophthalmol. 5, 1113-1118.

Zeng, Y., Guo, X., Lin, J., Zeng, X., Zhong, Y., Cai, X. and Liu, X. 2008. Effect of tear film changes on the 
intraocular measurement by Goldmann applanation tonometer. Yan. Ke. Xue. Bao. 24, 27-29.

Zhang. H., Yang, D., Ross, C.M., Wigg, J.P., Pandav, S. and Crowston, J.G. 2014. Validation of rebound tonometry for intraocular pressure measurement in the rabbit. Exp. Eye Res. 121, 86-93.
Zimmermann, M., Pitz, S., Schmidtmann, I., Pfeiffer, N. and Wasielica-Posiednik, J. 2017. Tonographic effect of ocular response analyzer in comparison to Goldmann applanation tonometry. Plos One. 12(1):e0169438.

10.1371/journal.pone.0169438. 